\title{
Personality and atrioventricular nodal reentrant tachycardia, beyond the topic
}

\author{
Irene Valverde André ${ }^{1}$ Julio Rodríguez Suárez ${ }^{2}$, \\ Mar González Vasserot ${ }^{1}$, David Calvo Cuervo ${ }^{3}$ \\ ${ }^{1}$ Electrophysiology Laboratory, Cardiology Department, Cabueñes Hospital, Los Prados, Gijon, Spain \\ ${ }^{2}$ Psychology Department, University of Oviedo, Oviedo, Spain \\ ${ }^{3}$ Hospital Universitario Central de Asturias, Instituto de Investigación Sanitaria \\ del Principado de Asturias, Oviedo, Spain
}

A general perception persists that patients with atrioventricular nodal reentry tachycardia (AVNRT) frequently show distinctive personality traits, attributed empirically to age (fifties) and gender (female), but no relevant studies have been performed in this setting. The present research aims to compare personality and temperamental type of patients with AVNRT and atrioventricular reentrant tachycardia (AVRT).

Consecutive patients from two hospitals were prospectively enrolled. Inclusion criteria consisted of electrocardiogram (ECG) confirming symptomatic supraventricular tachycardia and ablation acceptance. Exclusion criteria consisted of significant morbidity (including anxiety, depression, and personality disorders), treatment with benzodiazepines, serotonin inhibitors and beta-blockers or refusal to participate. The study was approved by the Ethics Committee on Human Research. All patients provided written informed consent. Eysenck Personality Inventory (EPI) was administered at the outpatient clinic before ablation and after a 6-month follow-up period.

Eysenck Personality Inventory [1] is comprised of 24 items for extraversion and 24 items for neuroticism. Each item is answered with 'yes' or 'no' and rated as either 0 or 1 . Total extraversion and neuroticism scores range from 0 to 24 points, with higher values representing greater extraversion and/or neuroticism. The extraversion is linked to the degree of excitation of the central nervous

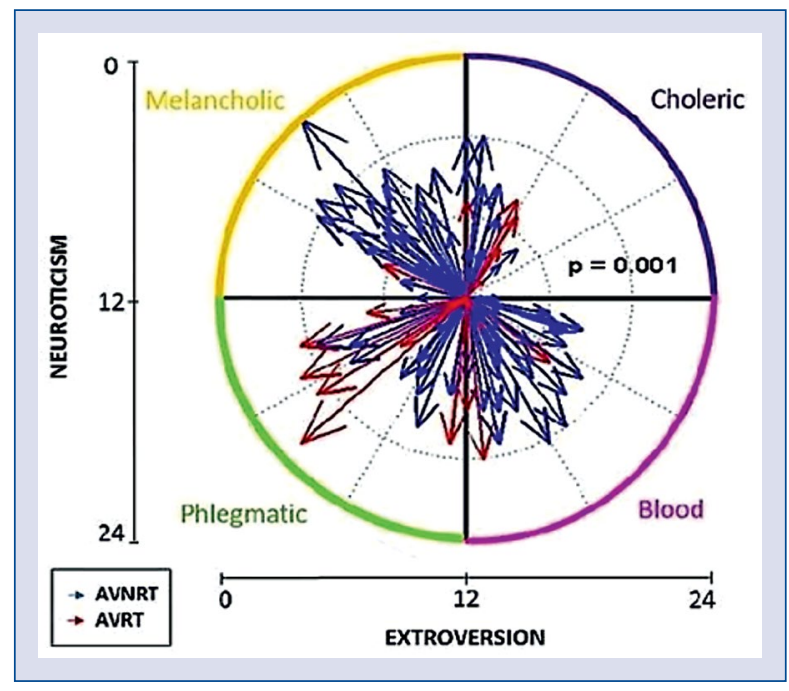

Figure 1. Vector conversion of temperamental types; atrioventricular nodal reentry tachycardia (AVNRT) patients in blue and atrioventricular reentrant tachycardia (AVRT) patients in red. AVRNT angle $13.1^{\circ}[95 \% \mathrm{Cl}$ $12^{\circ}$ to $\left.14^{\circ}\right]$ vs. AVRT angle $-34^{\circ}\left[95 \% \mathrm{Cl}-40^{\circ}\right.$ to $-28^{\circ}$; $\mathrm{p}=0.001 ; \mathrm{Cl}$ - confidence interval.

system and is defined as a compound of impulsivity and sociability. The neuroticism indicates the degree of excitation of the autonomic nervous system and is linked to being highly emotional.

Temperamental type was defined as the orthogonal intersection of extraversion and neuroticism axis (Fig. 1). In summary, sanguine is sociable

Address for correspondence: David Calvo Cuervo, MD, PhD, Hospital Universitario Central de Asturias, Instituto de Investigación Sanitaria, del Principado de Asturias, Oviedo, Spain, e-mail: davidcalvo307@gmail.com 
and the most extroverted; phlegmatic is peaceful and calm; melancholic is anxious, pessimistic and moody; and choleric is impulsive, optimistic and excitable [2]. For quantitative analysis, vectors were represented in a $360^{\circ}$ diagram andanalysed.

Variables were presented as mean and standard deviation (SD). The $\chi^{2}$, t-test and a general linear model were used when appropriate. Resampling methods (bootstrap) were used to compute $95 \%$ confidence intervals (95\% CI) and statistical significance was established at $\mathrm{p}<0.05$ (SPSSv23).

A total of 101 patients (median age 54 years; $68 \%$ women) were studied and divided into two groups according to supraventricular tachycardia diagnosis: AVNRT (81 patients) and AVRT (20 patients). No patient refused to participate. An electrophysiologic study and catheter ablation were successfully performed according to usual protocols in all patients, with no recurrences.

No differences in baseline characteristics (gender, age, tachycardia duration, time from first tachycardia episode to diagnosis, syncope related to tachycardia or attendance to the Emergency Department) were found.

Extraversion score in the AVNRT group was $12.45 \pm 4.17$ vs. $11.20 \pm 4.88$ in AVRT group $(\mathrm{p}=0.24)$, and the neuroticism score in the AVNRT group was $12.09 \pm 5.12$ vs. $10.40 \pm 5.03$ in the AVRT group $(\mathrm{p}=0.18)$.

Significant differences in melancholic temperamental type distribution (28.9\% in AVNRT vs. $5.3 \%$ in AVRT, $p=0.03$ ) were found between the groups by qualitative and quantitative analysis (Fig. 1).

Eysenck Personality Inventory was chosen to study personality for two main reasons. On the one hand, EPI evaluates personality from a simple structure: two orthogonal and bipolar factors, clearly defined and are considered the main factors in the study of personality, neuroticism and extroversion. On the other hand, EPI shows a very high reliability and validity, and are not only confined to a Spanish population [3].

In daily practice, it may be perceived that AVNRT patients, in contrast with AVRT patients, are often anxious, pessimistic, extroverted and demanding to such an extent that cardiologists colloquially talk about "the typical personality of AVNRT". This factor when applied to the present results, which could explain why observers perceive those differ- ences: among patients with AVNRT, melancholic temperamental type (anxious and pessimistic; the one with greater emotional lability) is frequent; however, among patients with AVRT, the melancholic type was the least frequent, and the most frequent were phlegmatic and choleric. This does not fit at all with the "AVNRT personality". Dysfunctional regulation of the hypothalamic-pituitary-adrenocortical system associated with melancholic temperament [4] could favour AVNRT physiology, but the exact mechanisms for this association remains unknown.

Extraversion and neuroticism decrease as people age, with the most pronounced declines occurring in young adulthood, but main personality changes in middle age are not expected [5]. Nevertheless, further research is needed to investigate whether being free from tachycardia episodes modulates personality.

According to the available research, distinctive melancholic temperamental type distribution between patients with AVNRT and AVRT is newly demonstrated. Further studies are needed to confirm these results.

\section{Acknowledgements}

To Beatriz Sampedro and all the colleagues in the Cardiology Departments.

\section{Conflict of interest: None declared}

\section{References}

1. Turet MS, Puig MF. de C. Spanish adaptation of the Eysenck EPI questionnaire. Anu Psicol UB J Psychol. 1972: 31-60.

2. Schmidt V, Firpo L, Vion D, et al. Eysenck's Psychobiological Personality Model: a projected story into the future. Rev Int Psicol. 2010: 11.

3. García Se, Pérez J, Tobeña A. Reliability and validity of the Spanish version of EPI. (Eysenck Personality Inventory). Rev Latinoam Psicol. 1979: 11.

4. Zobel A, Barkow K, Schulze-Rauschenbach S, et al. High neuroticism and depressive temperament are associated with dysfunctional regulation of the hypothalamic-pituitary-adrenocortical system in healthy volunteers. Acta Psychiatr Scand. 2004; 109(5): 392-399, doi: 10.1111/j.1600-0447.2004.00313.x, indexed in Pubmed: 15049775.

5. Milojev P, Sibley CG. Normative personality trait development in adulthood: A 6-year cohort-sequential growth model. J Pers Soc Psychol. 2017; 112(3): 510-526, doi: 10.1037/pspp0000121, indexed in Pubmed: 27831700. 